\title{
Anterior Chamber Foldable Intraocular Lens for Correction of Aphakia
}

\author{
Ashraf El Habbak ${ }^{1}$ and Mohamed Abdelzaher ${ }^{2 *}$ \\ 1Dar El Ouyon Hospital, Egypt \\ 2Benha University Hospital, Egypt
}

Submission: December 23, 2016; Published: February 28, 2017

*Corresponding author: Mohamed Abdelzaher, Benha University Hospital, Egypt, Email:Mohdzahir82.ma@gmail.com

\begin{abstract}
Purpose: To assess the outcomes of anterior chamber foldable intraocular lens (IOL) for aphakia correction.

Materials and methods: Retrospective non-comparative case series study for patients who underwent an operation for aphakia correction by the means of injection of an angular supported foldable IOL performed for 20 eyes of 15 patients. Student's paired t-test was carried out to compare pre-operative and post-operative visual acuity (VA), IOP \& corneal endothelial cell count.

Results: 15 patients were included in the study; 12 males \& 3 females. Mean age was $55.38 \pm 17.67$ years. Mean follow-up duration was $5.95 \pm 3.14$ months. The mean $\log$ MAR visual acuity was $1.26 \pm 0.46$ pre-operatively and $0.78 \pm 0.57$ post-operatively $(P=0.003)$. The change in IOP and corneal endothelial cell count were not statistically significant.
\end{abstract}

Conclusion: Injection of angular support foldable IOL in the AC is a useful technique for the correction of aphakia.

\section{Introduction}

Posterior capsular rupture is a well-known intra-operative complication of cataract surgery [1]. In complicated cases with insufficient capsule and lost zonular support it is not possible to insert a posterior chamber intraocular lens (PC IOL) [2]. Several techniques including posterior chamber sclera fixed lens, anterior chamber sulcus supported lens and anterior chamber iris claw lens have been reported to result in good visual out $\neg$ come in the management of aphakic eyes without capsular support [3]. The polymethylmetacrylate (PMMA) ante $\neg$ rior chamber (AC) intraocular lens has long been considered as a standard method of aphakia correction. This has been linked to various complications includ $\neg$ ing bullous keratopathy, increase of intra ocular pres $\neg$ sure (IOP), and endothelial cell loss or even visual loss [4]. Technological advances in lens design (angular support) and in lens mate $\neg$ rial (acrylic) have permitted the safe use of lens in the anterior chamber for refractive error correction $[4,5]$.

The aim of the current study was to determine the outcome of using the anterior chamber (AC) foldable lens for unilateral aphakia.

\section{Materials and Methods}

This is a retrospective non-comparative, consecutive case series study, in which 20 eyes of 15 patients underwent an operation for aphakia correction by the means of injection of angular support foldable intraocular lens in the anterior chamber between January 2014 and December 2014 in Dar El Ouyon Hospital. Written informed consent was obtained after explaining the nature of the procedure from all patients before surgery. Preoperatively, each patient underwent a detailed ocular history (indicating the cause of aphakia) and a standard eye examination including testing of uncorrected and best corrected distance visual acuity (BCVA) using Snellen visual acuity chart (converted into log MAR units for statistical purposes) measurement of the intra ocular pressure (IOP) by Goldmann applanation tonometer, slit lamp examination focusing on the corneal details, and gonioscopy, fundus, and retinal periphery examination. Biometry, anterior chamber depth and corneal White-to-White (WTW) diameter were measured using the IOL Master 500 (Carl Zeiss Meditec AG). Specular microscopy was done to reveal endothelial cell count and abnormal forms using inclusion criteria were unilateral or bilateral aphakia 
with no capsular support or clinical keratopathy, no evidence of glaucoma, open iridocorneal angle, AC depth $>3 \mathrm{~mm}$, WTW diameter $>11.5 \mathrm{~mm}$, and improvement of the visual acuity with corrective trial lenses.

Patients with clinical keratopathy, glaucoma, uveitis, peripheral anterior synechia or clinical maculopathy were excluded. All surgeries were conducted under local peri-bulbar anesthesia using Bupivacaine 0.5\% + Xylocaine 2\%. One 1-mm paracentesis was made at the upper nasal position and one $2.80 \mathrm{~mm}$ clear corneal tunnel incision was made at the upper temporal position with the keratome. A viscoelastic (Viscoat, Alcon) substance was used to reform the AC. The foldable single piece acrylic intraocular lens with the angular support was then injected gently in the anterior chamber, (Figure 1\&2). A peripheral iridectomy was done using Vanas scissors. The viscoelastic substance was removed from the anterior chamber with the Simcoe cannula. At the end of the procedure, a subconjonctival depot of dexamethazone and gentamicin was given.

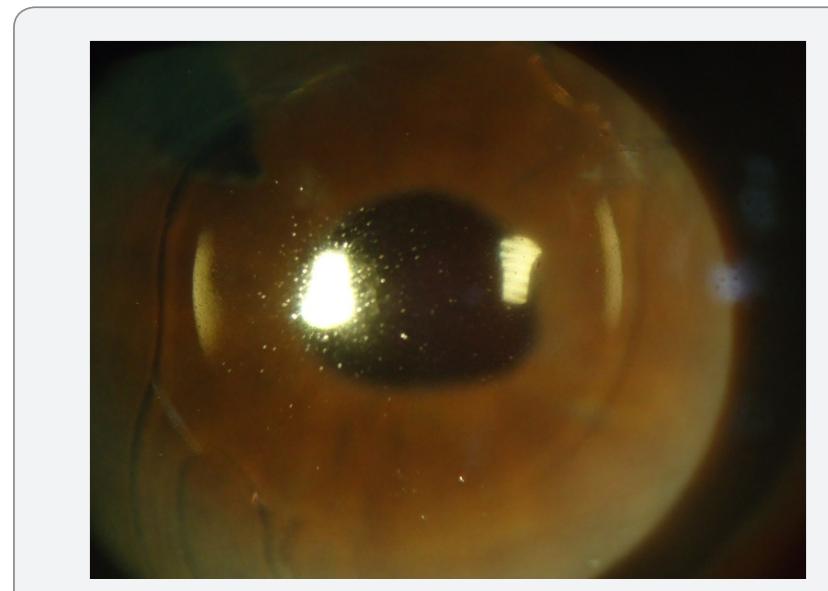

Figure 1: Shows loading of the foldable anterior chamber IOL.

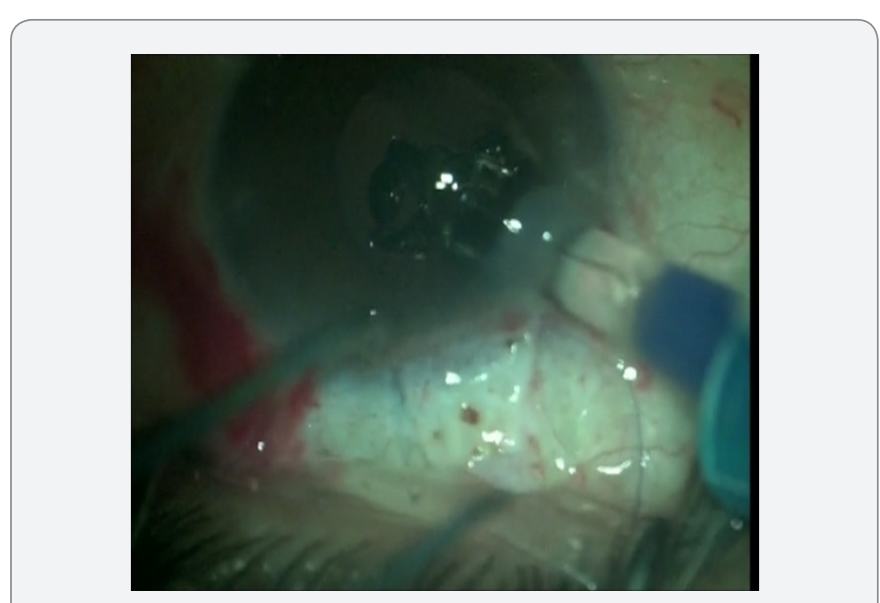

Figure 2: Shows injection of the foldable anterior chamber IOL.

Follow-up visits were scheduled as follows: one day, one week, two months, six months, and one year following surgery. At each follow-up visit, BCVA and IOP were assessed and corneal endothelial cell count and morphology were analyzed. Figure
(3) shows foldable anterior chamber IOL in place. Student's paired t-test was carried out to compare post-operative and pre-operative visual acuity (VA) and intraocular pressure (IOP). The analysis was performed using statistical software Epi-Info 7 and IBM SPSS 19. P-values less than 0.05 were considered statistically significant.

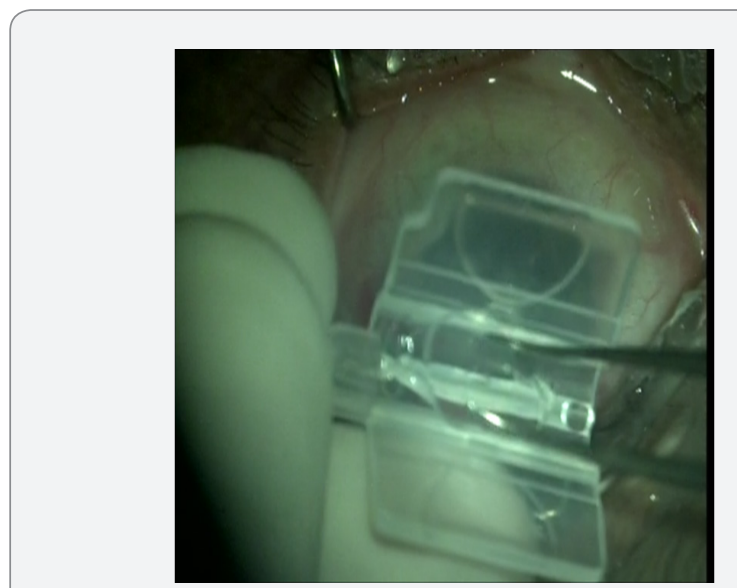

Figure 3: Foldable anterior chamber IOL in place.

\section{Results}

Twenty (20) eyes of fifteen (15) patients were included in this study. The demographics and baseline eye characteristics for all patients are presented in . Eight patients were males (10 eyes, $50 \%$ ) and seven were females (10 eyes, 50\%). Mean age was $50.75 \pm 11.275$ years (range $30-72$ years). Aphakia causes included: 2 planed ICCE (10\%), 6 inadvertent posterior capsule rupture with vitreous lost during planed phacoemulsification (30\%) and 12 post-traumatic lens subluxation (60\%). The mean follow-up duration was $(10.8 \pm 2.02)$ months (range 6-12 months). Sixteen $(80 \%)$ patients showed improvement of the post-operative BCVA of one line or more; two (10\%) showed no improvement and two (10\%) patients showed deterioration in their post-operative BCVA. The mean visual acuity in log MAR was $(1.14 \pm 0.46)$ pre-operative and $(0.605 \pm 0.489)$ post-operative ( $\mathrm{P}<0.05)$. Post-operative complications are presented in. Two patients $(10 \%)$ had post-operative intraocular hypertension (over $21 \mathrm{mmHg}$ ). Two (20\%) patients had pupil distortion, one patient $(10 \%)$ had retinal detachment and one $(10 \%)$ patient had macular edema. No corneal problem requiring lens removal was registered.

\section{Discussion}

There are many options for management of aphakia with no capsular support [5]. Anterior chamber IOL is one of these options. Foldable lenses with angular support are currently used in the anterior chamber for refractive error correction [6]. In this study, we injected a foldable lens with angular support in the anterior chamber to correct the aphakia. All patients presented with aphakia without capsular support from various etiologies. Post-traumatic lens subluxation was the leading cause of aphakia $(60 \%)$ in our study. Sixteen $(80 \%)$ patients showed significant 
improvement of the BCVA from $(1.14 \pm 0.46)$ pre-operative to $(0.605 \pm 0.489)$ post-operative $(\mathrm{P}<0.05)$. Previous studies using an iris claw lens to correct aphakia reported significant VA improvement and a low rate of complications [5,7]. However, despite the good visual outcome, implementation of iris claw techniques requires specialized equipment and a long training period. The insertion of a foldable lens in anterior chamber is a very easy procedure through a $2.80-3 \mathrm{~mm}$ clear corneal tunnel.

In our study, 2 eyes (10\%) developed of post operatively a transient IOP elevation, which was managed with topical medications. This change in IOP was not statistically significant $(\mathrm{P}=0.32)$. It was also reported in other similar studies [13] in which other approaches were used to correct aphakia. Two cases $(10 \%)$ with pupil distortion were observed postoperative, which was also noted, in another study (2). One case (5\%) developed macular edema post-operative, which resolved on topical steroids and non-steroidal anti-inflammatory drops. One case (5\%) developed post-operative retinal detachment. A vitrectomy and silicone oil injection was done. These two complications resulted in lower VA in our study.

\section{Conclusion}

Our results showed that foldable IOL injection in the anterior chamber improved visual acuity; the complications were similar to those reported in the literature using other methods. Although the visual outcome was good for most patients in this series, more extended follow up and a large series of patients are needed to ascertain the effectiveness and safety of this procedure.

\section{What was Known}

Rigid anterior chamber IOLs were commonly used for visual rehabilitation in cases of aphakia. This was associated with many complications such as elevated intraocular pressure, anterior uveitis and corneal decompensation.

\section{What this Paper Adds}

Using foldable anterior chamber IOL provides easy, safe and effective method for correction of aphakia.

\section{References}

1. Kukner AS, Alagoz G, Erdurmus M, Serin D, Dogan U, et al. (2014) Anterior chamber fixation of a posterior chamber intraocular lens: A novel technique. Indian J Ophthalmol 62(4): 487-489.

2. Fasih U, Ahmed I, Shaikh A, Fahmi MS (2010) Comparison of Complications after Primary and Secondary Anterior Chamber Intraocular Lens Implantation. Pak J Ophthalmol (26)2.

3. Giles K, Ernest M, Christelle D, Georges NT, Raoul C, et al. (2013) Aphakia Correction by Injection of Foldable Intra Ocular Lens in The Anterior Chamber. Ophthalmol Eye Dis (5) 17-22.

4. Yadav NK, Kemmanu V, Bhargava M, Shetty B (2012) A truly knotless technique for scleral fixation of intraocular lenses: two-year results. Indian J Ophthalmol 60(2): 147-148.

5. De Silva SR, Arun K, Anandan M, Glover N, Patel CK, et al. (2011) Irisclaw intraocular lenses to correct aphakia in the absence of capsule support. J Cataract Refract Surg 37(9): 1667-1672.

6. AkcayL, Eser I, Kaplan AT, Taskiran Comez A, Dogan OK (2012) Phakic anterior chamber lenses in very high myopia: an 18-month follow up. Clin Experiment Ophthalmol 40(3): 275-281.

7. Gonnermann J, Klamann MK, Maier AK, Rjasanow J, Joussen AM, et al. (2012) Visual outcome and complications after posterior iris-claw aphakic intraocular lens implantation. J Cataract Refract Surg 38(12): 2139-2143.

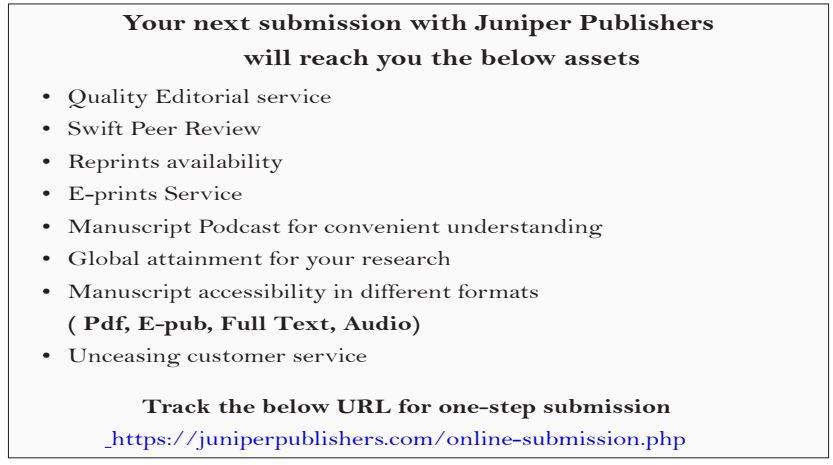

